\title{
Research Progress on Ni-Based Antiperovskite Compounds
}

\author{
P. Tong ${ }^{1}$ and Y. P. Sun ${ }^{1,2}$ \\ ${ }^{1}$ Key Laboratory of Materials Physics, Institute of Solid State Physics, Chinese Academy of Sciences, Hefei 230031, China \\ ${ }^{2}$ High Magnetic Field Laboratory, Chinese Academy of Sciences, Hefei 230031, China
}

Correspondence should be addressed to Y. P. Sun, ypsun@issp.ac.cn

Received 19 September 2012; Accepted 5 December 2012

Academic Editor: Laifeng Li

Copyright ( 2012 P. Tong and Y. P. Sun. This is an open access article distributed under the Creative Commons Attribution License, which permits unrestricted use, distribution, and reproduction in any medium, provided the original work is properly cited.

The superconductivity in antiperovskite compound $\mathrm{MgCNi}_{3}$ was discovered in 2001 following the discovery of the superconducting $\mathrm{MgB}_{2}$. In spite of its lower superconducting transition temperature ( $8 \mathrm{~K}$ ) than $\mathrm{MgB}_{2}(39 \mathrm{~K}), \mathrm{MgCNi}_{3}$ has attracted considerable attention due to its high content of magnetic element $\mathrm{Ni}$ and the cubic structure analogous to the perovskite cuprates. After years of extensive investigations both theoretically and experimentally, however, it is still not clear whether the mechanism for superconductivity is conventional or not. The central issue is if and how the ferromagnetic spin fluctuations contribute to the cooper paring. Recently, the experimental results on the single crystals firstly reported in 2007 trend to indicate a conventional $s$-wave mechanism. Meanwhile many compounds neighboring to $\mathrm{MgCNi}_{3}$ were synthesized and the physical properties were investigated, which enriches the physics of the Ni-based antiperovskite compounds and help understand the superconductivity in $\mathrm{MgCNi}_{3}$. In this paper, we summarize the research progress in these two aspects. Moreover, a universal phase diagram of these compounds is presented, which suggests a phonon-mediated mechanism for the superconductivity, as well as a clue for searching new superconductors with the antiperovskite structure. Finally, a few possible scopes for future research are proposed.

\section{Introduction}

To explore new superconductors is one of the central issues of material science and condensed matter physics. The discovery of high-temperature (high- $T_{C}$ ) superconductivity in cuprates has attracted a lot of attention in the past decades [1]. In 2001, Professor R. J. Cava from the University of Princeton reported the superconductivity in antiperovskite compound $\mathrm{MgCNi}_{3}$ with the transition temperature $T_{C} \sim$ $8 \mathrm{~K}$ (Figure 1) [2]. The superconductivity in $\mathrm{MgCNi}_{3}$ is unusual in view of the large content of the magnetic element $\mathrm{Ni}$, which often favors a magnetic ground state. A prominent feature of the electronic structure is an extended van Hove singularity as shown in Figure 2(a), giving rise to a large density of states (DOS) just below the Fermi level $\left(E_{F}\right)$ [see Figure 2(b)] [3]. A similar feature has been observed in some high- $T_{C}$ superconductors. Moreover, the DOS peak is mainly attributive to the Ni $3 \mathrm{~d}$ states $[4,5]$. Structurally, the cubic symmetry recalls the high- $T_{C}$ cuprates superconductors with perovskite structures. Thus the central question was raised, whether the superconductivity in $\mathrm{MgCNi}_{3}$ is exotic. In other words, the answer to the question lies in clarifying the roles of the spin fluctuations or ferromagnetic (FM) correlations probably from the dominant $\mathrm{Ni}$ content in $\mathrm{MgCNi}_{3}$. However, the experimental results based on polycrystalline samples by different techniques (such as NMR [6], London penetration depth $[7,8]$, critical current behavior [9], tunneling spectra $[10,11]$, carbon isotope effect [12], specific heat $[13,14], \mu \mathrm{SR}[15]$ and so on) from different groups are controversial. A detailed summary on the experimental and theoretical results published before 2004 can be found in the review paper [16] written by Mollah. From then on, the researchers have been focusing on two main scopes in this field, namely, the experimental investigations on $\mathrm{MgCNi}_{3}$ single crystals and on the synthesis and physical properties of neighbor compounds of $\mathrm{MgCNi}_{3}$, which have never been included in any review papers. In this paper, we focus on these two topics, as well as give a phase diagram based on the available data of the lattice constant, the Debye temperature and the density of state at the Fermi level, $N\left(E_{F}\right)$, for the Ni-based antiperovskite compounds. The phase diagram supports that the superconductivity observed in the Ni-based 


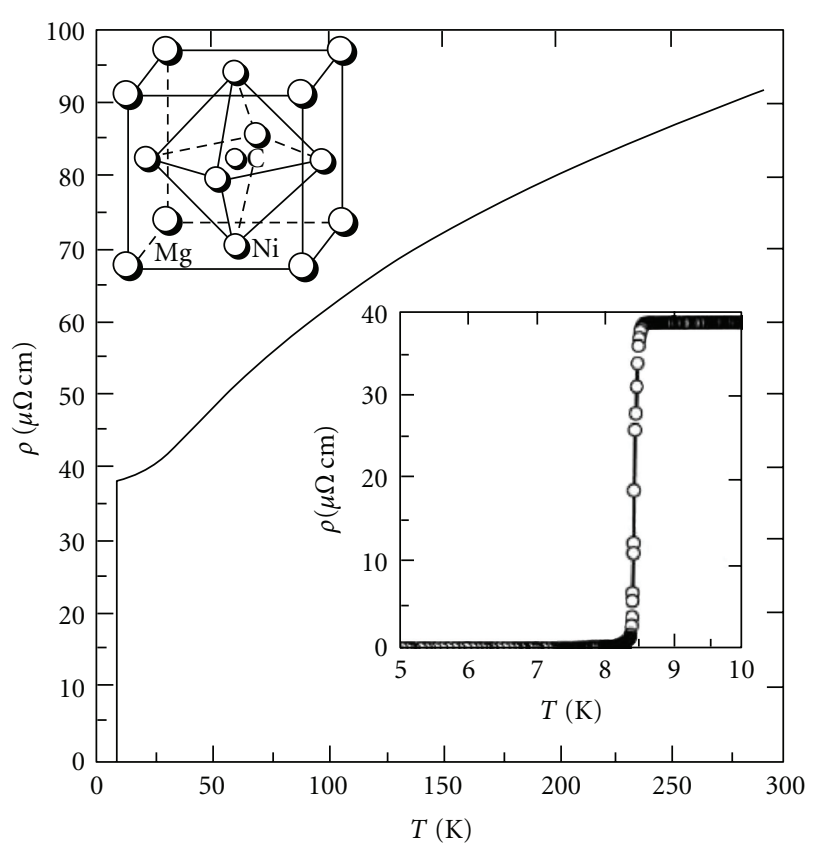

FIGURE 1: The temperature-dependent resistivity for polycrystalline $\mathrm{MgCNi}_{3}$ [Adapted by permission from Macmillan Publishers Ltd: Nature. He et al., Nature, 411, 6833 (2001), copyright 2001]. The up and low insets show the antiperovskite crystal structure and superconducting transition, respectively.

antiperovskite compounds is rather phonon-mediated than unconventional. The phase diagram also helps explore new superconductors in Ni-based antiperovskite compounds. Some possible future research scopes were proposed in the end of this paper.

\section{Experimental Results on Single Crystalline $\mathrm{MgCNi}_{3}$}

The experiments on single crystal are desirable for eliminating the discrepancies in the experimental results based on polycrystalline samples. However, the first, also the only successful synthesis of $\mathrm{MgCNi}_{3}$ single crystal up to date, was reported in 2007 [17] by Lee et al., five years after the discovery of superconductivity in polycrystalline $\mathrm{MgCNi}_{3}$.

In [17], Lee et al. employed a self-flux method with the aid of high pressure. The mixtures of $\mathrm{Mg}, \mathrm{C}$, and Ni powders with the ratio $1: 1: 3$ were ground, pressed into a pellet, and then loaded into a high pressure cell. Then the sample was heated at $1200^{\circ} \mathrm{C}$ under $4.25 \mathrm{GPa}$ for 12 hours. The resulted sample is a mixture of single crystalline $\mathrm{MgCNi}_{3}$ with the size of hundreds of micrometers and some fluxes. Unlike the polycrystalline samples, the single crystal does not contain C or $\mathrm{Mg}$ deficiencies. Instead, $\mathrm{Ni}$ is found to be deficient. The real composition turns out to be $\mathrm{MgCNi}_{2.8 \pm 0.05}$. As displayed in Figure 3, the transition temperature is found to be $6.7 \mathrm{~K}$, slightly lower than the $T_{C}$ for polycrystalline $\mathrm{MgCNi}_{3}$. Even so, the entire sample quality was greatly improved compared with the crystalline samples. For example, the
TABLE 1: The lattice constant $a$, superconducting transition temperature $T_{C}$, low critical field $H_{C 1}(0)$, up critical field $H_{C 2}(0)$, coherence length $\xi(0)$, penetration depth $\lambda(0)$, Ginzgurg-Landau parameter $\kappa(0)$, electronic specific heat coefficient (Sommerfeld constant) $\gamma$, Debye temperature $\Theta_{D}$, coupling ratio $2 \Delta / k_{B} T_{C}$, and the density of state at the Fermi level $E_{F}, N\left(E_{F}\right)$, for the Ni-based antiperovskite superconductors. The parameters are mainly from [41]. The parameters under the "/" are for $\mathrm{MgCNi}_{3}$ single crystal from [17-20]. The values of $N\left(E_{F}\right)$ are from theoretical calculations reported in $[4,28,42]$.

\begin{tabular}{lccc}
\hline & $\mathrm{MgCNi}_{3}$ & $\mathrm{CdCNi}_{3}$ & $\mathrm{ZnNNi}_{3}$ \\
\hline$a(\AA)$ & $3.812 / 3.8125$ & 3.844 & 3.756 \\
$T_{C}(\mathrm{~K})$ & $7.6 / 7.3$ & 3.2 & 3 \\
$H_{C 1}(0)(\mathrm{mT})$ & 10 & 8.6 & 6.9 \\
$H_{C 2}(0)(\mathrm{T})$ & $14.4 / 12.8$ & 2.2 & 0.96 \\
$\xi(0)$ & $46 / 51$ & 122 & 185 \\
$\lambda(0)(\AA)$ & $2480 / 2300$ & 2767 & 3089 \\
$\kappa(0)$ & $54 / 44$ & 23 & 17 \\
$\gamma\left(\mathrm{mJ} \cdot \mathrm{mol}^{-1} \mathrm{~K}^{-2}\right)$ & 30.1 & 18 & 13 \\
$\Theta_{D}(\mathrm{~K})$ & $284 / 132$ & 352 & 336 \\
$2 \Delta / k_{B} T_{C}$ & $(3.75-5) / 4$ & - & - \\
$N\left(E_{F}\right)$ & 4.99 & 3.82 & 2.813 \\
$($ states eV & & & \\
\hline
\end{tabular}

residual resistivity ratio is 2.7 , larger than the values ever reported for the crystalline samples. Moreover, the single crystal was homogeneous and free of microscopic regular arrays observed in the high-resolution transmission electron microscopy (TEM) images for polycrystalline samples [17].

In order to clarify the nature of the superconductivity in single crystal $\mathrm{MgCNi}_{3}$, further measurements have been performed on the samples from Lee's group. Based on the resistance measured as functions of the temperature and the applied magnetic field, it is found that the normal state resistivity can be explained by using only electron-phonon (e-p) scattering mechanism, indicating a conventional BCS behavior [18]. It is further supported by the linear behavior of $H_{C 2}(T)$ near $T_{C}$. The low-temperature electronic specific heat $C_{p}(T)$ in superconducting state shows a classical exponential decrease confirming $s$-wave pairing with a moderate e-p coupling in this material $[19,20]$. However, the $C_{p}(T)$ at normal state cannot be well described by the usual $T^{3}$ term of phonon contribution. A higher phonon-term probably due to the softening of the lowest acoustic Ni phonon modes is needed to interpret the deviation [20]. It is consistent with the magnetic penetration depth measured by highprecision tunnel diode oscillator technique and Hall probe magnetization, which shows that the superconducting gap is fully open over the whole Fermi surface [21]. Moreover, the ratio $2 \Delta / k_{B} T_{C} \approx 4$ and high specific-heat jump at $T_{C}$ in zero field, $\Delta \mathrm{C}\left(T_{C}\right) / \gamma_{n} T_{C} \approx 1.96$, indicating a strongcoupling mechanism. This scenario is supported by the direct gap measurements via the point-contact spectroscopy [20]. The reported superconducting parameters are summarized in Table 1. The availability of single crystal specimens also allows a detailed phonon-dispersion mapping which is 


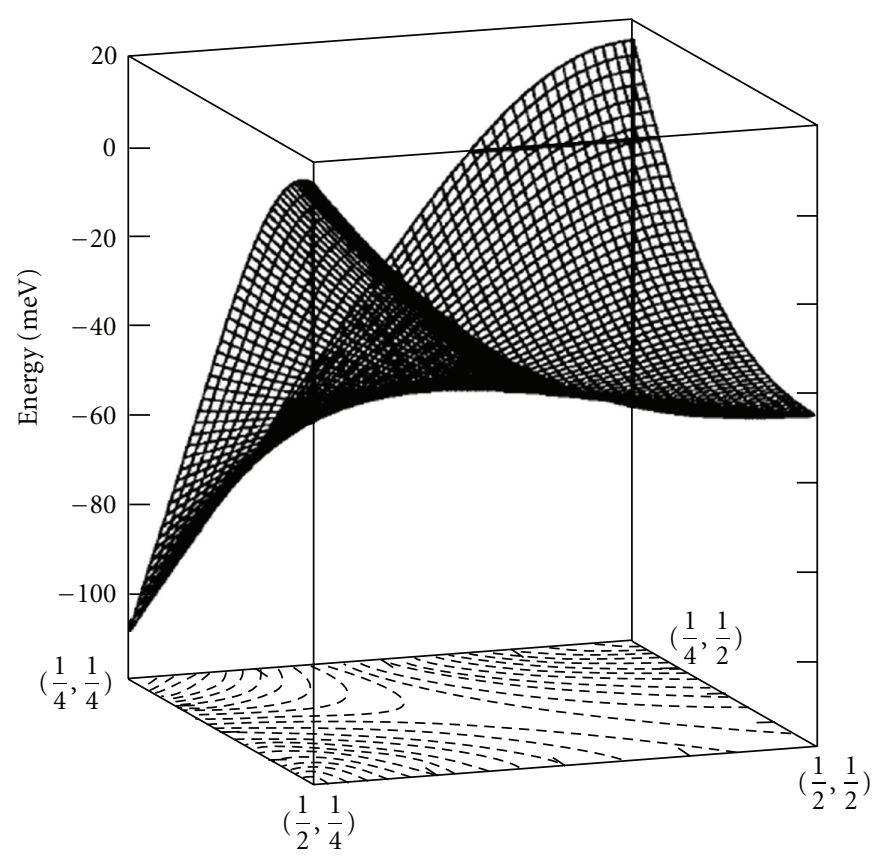

(a)

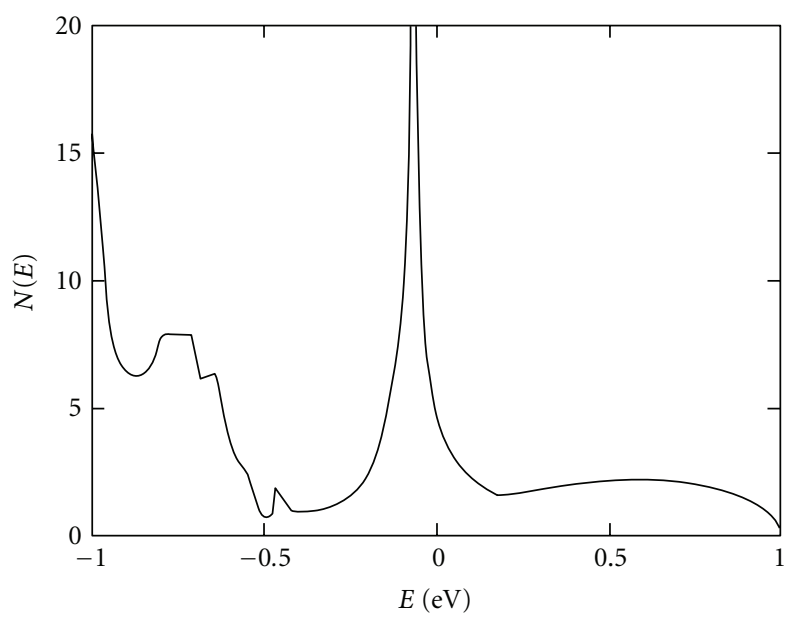

(b)

FIgure 2: (a) Surface plot (and contour plot below) of the van Hove singularity in $\varepsilon_{k}$ (relative to $E_{F}$ ) in the G-M-X plane, with $M$ at the right-hand corner (planar coordinates are given in units of $2 \pi / a$ ) [Reprinted with permission from H. Rosner et al., Phys. Rev. Lett. 88, 027001 (2002)]. (b) The peak structure in the electronic density of state around $E_{F}$ [Reprinted with permission from D. J. Singh et al., Phys. Rev. B 64, 140507 (2001)].

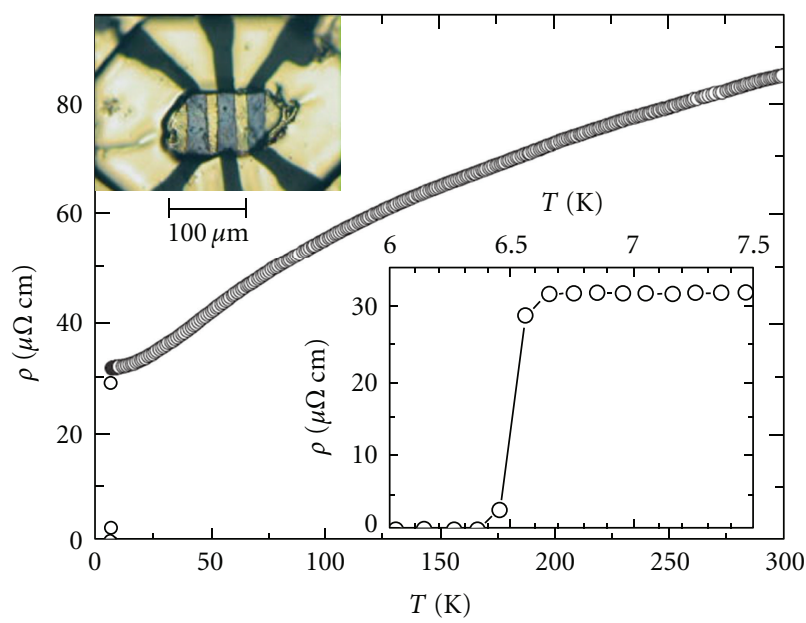

Figure 3: (Color online) Temperature-dependent resistivity of $\mathrm{MgCNi}_{2.8}$ [Reprinted with permission from H. -S. Lee et al., Adv. Mater. 19, 1807-1809 (2007)]. The sample with four metallic leads is shown in the upper inset. The lower inset shows a magnified view of $\rho(T)$ near the superconducting transition.

closely related to the superconducting mechanism. By applying inelastic X-ray scattering (IXS), the phonon mapping was reported by Hong et al. [22]. The IXS result implies that there are no phonon anomalies that could support any exotic mechanisms for superconductivity in $\mathrm{MgCNi}_{3}$. This result was verified by a late $a b$ initio calculation [23]. In addition, Jang et al. [24] observed the collapse of the peak effect $(\mathrm{PE})$, namely a sudden increase in the critical current near the end of superconductivity. As the AC driving frequency increases, the PE was collapse and observable flux creep was developed in contrast to the result observed in the wellstudied $\mathrm{NbSe}_{2}$. Also, the $\mathrm{PE}$ in $\mathrm{MgCNi}_{3}$ was suggested be a dynamic phenomenon.

Although the experimental results measured on the single crystal samples suggest that $\mathrm{MgCNi}_{3}$ is a conventional BCS-type superconductor with mediate or strong e-p coupling, it is yet arbitrary to exclude the contribution from spin fluctuations or FM instability. The reason is relative to the single crystalline sample itself. All the experiments were performed on the crystals prepared by the same group. Moreover, the crystal is Ni-deficient [17] though its superconducting parameters are close to those determined on polycrystalline samples (see Table 1). Theoretically, for another Ni-based antiperovskite compound $\mathrm{InCNi}_{3}$, it is proved that the excess of $\mathrm{Ni}$, or say, deficiency of In can tune the system to the FM instability [25], even to a FM order [26]. It is natural to expect stronger spin-fluctuations given a perfectly stoichiometric $\mathrm{MgCNi}_{3}$ single crystal. Therefore, a theoretical comparison between the Ni-deficient $\mathrm{MgCNi}_{3-\delta}$ and perfect $\mathrm{MgCNi}_{3}$ would resolve the problem. Moreover, growth of single crystals without $\mathrm{Ni}$ deficiencies is needed to end the ten-year debate on whether $\mathrm{MgCNi}_{3}$ is unconventional superconductor. 


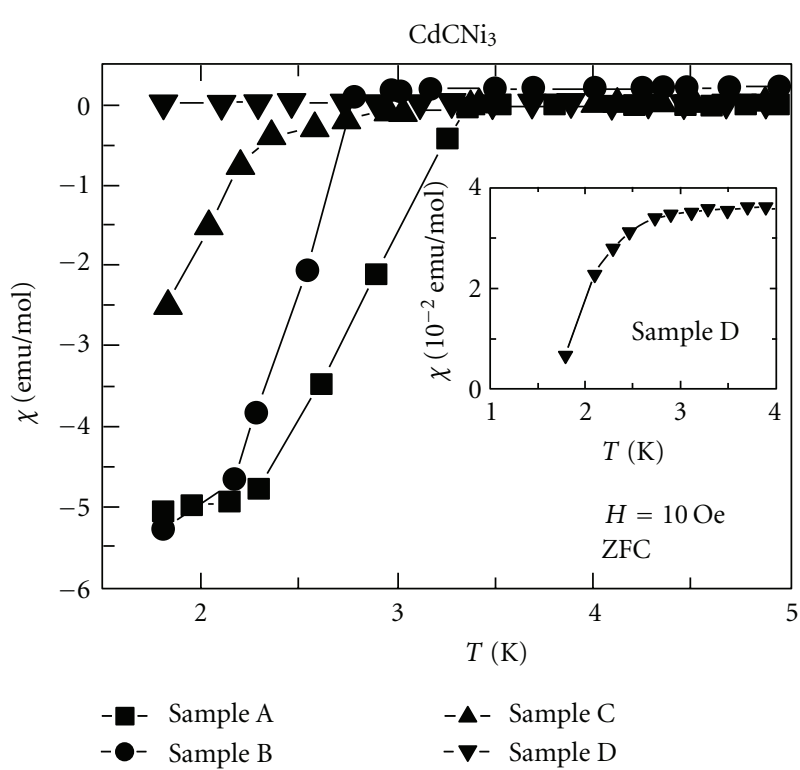

(a)

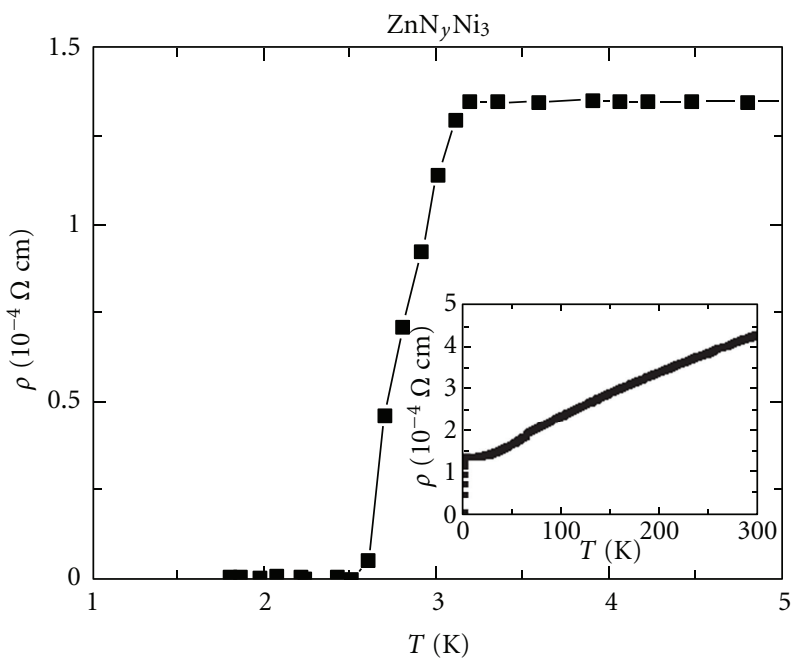

(c)

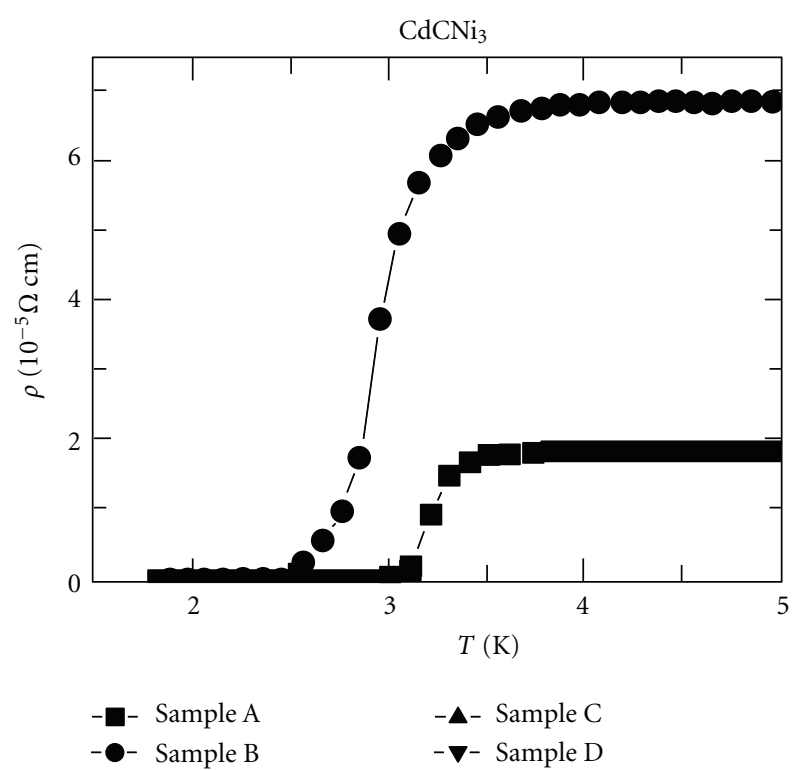

(b)

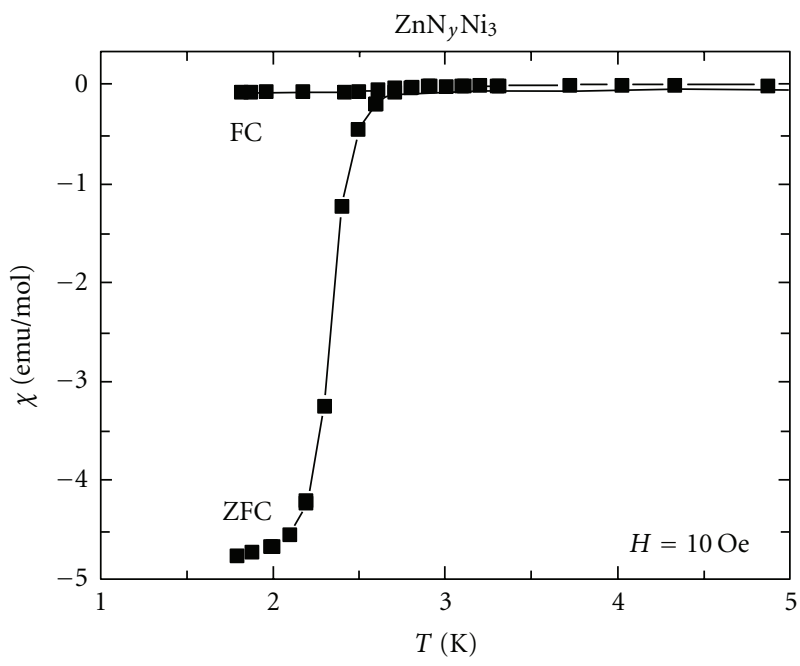

(d)

FIGURE 4: Temperature dependence of magnetic susceptibility (a) and electrical resistivity (b) for different CdCNi $\mathrm{Camples}_{3}$ [Reproduced with permission from M. Uehara et al., J. Phys. Soc. Jpn. 76, 034714 (2007)]. Temperature dependence of electrical resistivity (c) and magnetic susceptibility (d) for $\mathrm{ZnN}_{y} \mathrm{Ni}_{3}$ [Reproduced with permission from M. Uehara et al., J. Phys. Soc. Jpn. 78, 033702 (2009)]

\section{Research Progress on Ni-Based Antiperovskite Compounds other than $\mathrm{MgCNi}_{3}$}

The purpose of investigating the materials which are closely related to $\mathrm{MgCNi}_{3}$, that is, $\mathrm{AXNi}_{3}(\mathrm{~A}=\mathrm{Zn}, \mathrm{Al}, \mathrm{Ga}$, In, $\mathrm{Cd}$ and so on; $\mathrm{X}=\mathrm{C}, \mathrm{N}, \mathrm{B}$ ), is two sided to explore new superconductors and to shed light on the superconducting mechanisms for $\mathrm{MgCNi}_{3}$. Up to date, there are more than ten compounds neighboring to $\mathrm{MgCNi}_{3}$ were synthesized and the physical properties investigated. These newly synthesized Ni-based antiperovskite compounds can be grouped into three types, that is, carbides $\mathrm{ACNi}_{3}$, nitrides $\mathrm{ANNi}_{3}$, and borides $\mathrm{ABNi}_{3}$.
$\mathrm{CdCNi}_{3}$ with the same number of valence electrons as $\mathrm{MgCNi}_{3}$ is another superconductor in the carbides $\mathrm{ACNi}_{3}$. As shown in Figures 4(a) and 4(b), the transition temperature $T_{C}$ is around $3.2 \mathrm{~K}$, varying with fabrication conditions [27]. The superconducting parameters are listed in Table 1. The specific heat Sommerfeld constant $\gamma$ is $18 \mathrm{~mJ} /\left(\mathrm{mol} \mathrm{K}^{2}\right)$, smaller than that of $\mathrm{MgCNi}_{3}$. However, the theoretical calculation shows the $N\left(E_{F}\right)$ value is slightly larger than $\mathrm{MgCNi}_{3}$, while the calculated e-p coupling coefficient (0.8) is nearly half that of the corresponding value of 1.5 for $\mathrm{MgCNi}_{3}$ [28]. This is argued to be associated with a softening behavior of the lowest acoustic phonon branch along the X$\mathrm{R}$ symmetry direction [28]. The large Wilson ratio $R_{W}=$ $\left(\pi^{2} \kappa_{B}^{2} / 3 \mu_{B}^{2}\right)\left(\chi_{0} / \gamma\right) \sim 12$ and the well suppressed upper critical 
field $H_{c 2}(0) \sim 2.2 \mathrm{~T}$, compared with the Pauli limit $(14 \mathrm{~T})$ indicate the existence of strong FM correlations. Surprisingly, $\mathrm{ZnCNi}_{3}$ with the same number of valence electrons, as $\mathrm{MgCNi}_{3}$ and $\mathrm{CdCNi}_{3}$, is found to be a Pauli paramagnetic (PM) metal without signals of superconductivity down to $2 \mathrm{~K}$ [29]. The value of $\gamma$ is only $6.77 \mathrm{~mJ} /\left(\mathrm{mol} \mathrm{K}^{2}\right)$, much smaller than those of $\mathrm{MgCNi}_{3}$ and $\mathrm{CdCNi}_{3}$ (see Table 1), indicating a very weak e-p coupling that explains the disappearance of superconductivity. However, it was theoretically suggested that the experimental $\mathrm{ZnCNi}_{3}$ is carbon deficient, while the stoichiometric compound should be superconducting [30].

The polycrystalline $\mathrm{ACNi}_{3}(\mathrm{~A}=\mathrm{Al}, \mathrm{Ga}, \mathrm{In})$ series with one more valence electron than $\mathrm{MgCNi}_{3}$ were prepared by solid state reaction and detailed studies of their basic properties were performed. For $\mathrm{GaCNi}_{3}$, a $T^{2}$ temperature dependence of resistivity was observed. The large values of the Kadowakiwoods ratio $A / \gamma^{2} \sim 7.2\left(\mu \Omega \mathrm{cm} / \mathrm{K}^{2}\right)$ and the Wilson ratio $R_{W} \sim 9.2$ suggest a highly correlated Fermi liquid behavior [31]. The large electron-electron correlation was suggested to be caused by the proximity of FM order from the side of exchange-enhanced Pauli paramagnet, evidenced by the remarkable enhancements in both the specific heat Sommerfeld constant $\gamma$ and the temperature-independent magnetic susceptibility $\chi_{0}$. As to $\mathrm{AlCNi}_{3}$ compound, the magnetic properties also show it is a strongly exchangeenhanced Pauli paramagnet in the very vicinity of FM order [32]. However, the low-temperature resistivity is nearly linear temperature-dependent, indicating a possible non-Fermiliquid behavior which is in sharp contrast with $\mathrm{GaCNi}_{3}$. The low-temperature electronic specific heat reveals that the spin fluctuations in $\mathrm{AlCNi}_{3}$ are strongly enhanced when compared with the superconducting $\mathrm{MgCNi}_{3}$, while the e-p couplings are comparable in both compounds. The Wilson ratio $R_{W}$ is about 2.4 and the dimensionless ratio that connects the low-temperature Seebeck coefficient with the Sommerfeld specific heat constant indicate that $\mathrm{AlCNi}_{3}$ can be considered as a modest electron-correlated material. Consistently, the enhanced spin fluctuations were confirmed using ${ }^{27} \mathrm{Al}$ NMR measurement in $\mathrm{AC}_{x} \mathrm{Ni}_{3}$ with $x \geq 0.1$ where the FM order was suppressed and the system is in the vicinity of FM order [33, 34]. On the contrary, an early experimental report shows $\mathrm{AlCNi}_{3}$ is a weak ferromagnet with the FM$\mathrm{PM}$ transition at $300 \mathrm{~K}$ [35]. The nonmagnetic ground state for $\mathrm{AlCNi}_{3}$ and $\mathrm{GaCNi}_{3}$ was confirmed by many theoretical reports [36-39], though the predicted FM correlations or spin fluctuations are weaker than experimentally measured [37]. The existence of carbon deficiencies to various extents may account for this divergence, as suggested by Sieberer et al. [37]. As to the $\mathrm{InCNi}_{3}$, it was found that the reduction of Indium ratio in the mixture of the raw powders helps make pure antiperovskite type compound [26]. The resulted composition from the optimum synthesis is $\operatorname{In}_{0.95} \mathrm{CNi}_{3}$. It behaves as a FM metal below the Curie temperature (577 K) [26]. It was suggested that the appearance of ferromagnetism originates from the deviation of the $\mathrm{Ni} / \mathrm{In}$ atomic ratio from the ideal case. Theoretically, it is found the ideally stoichiometric $\mathrm{InCNi}_{3}$ is a nonmagnetic metal and far away from a long-range magnetic order $[25,40]$. Both In vacancies and substitutional $\mathrm{Ni}$ on In site were found to be able to lead to a spin-polarized ground state. Energetically, the latter scenario is more preferable to generate a FM ground state [25].

$\mathrm{ZnNNi}_{3}$ is the only superconductor observed so far in the nitrides $\mathrm{ANNi}_{3}$ [41]. The $T_{C} \sim 3 \mathrm{~K}$, as shown in Figures $4(\mathrm{c})$ and $4(\mathrm{~d})$, is close to that of $\mathrm{CdCNi}_{3}$. The magnetic susceptibility shows a Pauli-like behavior with the magnitude much smaller than that of $\mathrm{CdCNi}_{3}$. It indicates the FM correlations in this material are not as enhanced as in $\mathrm{CdCNi}_{3}$. The obtained specific heat Sommerfeld constant $\gamma$ is $13 \mathrm{~mJ} /\left(\mathrm{mol} \mathrm{K}^{2}\right)$, smaller than the value of $18 \mathrm{~mJ} /\left(\mathrm{mol} \mathrm{K}^{2}\right)$ for $\mathrm{CdCNi}_{3}$. Even so, the $T_{C}$ is close to $\mathrm{CdCNi}_{3}$ because the FM correlation which could suppress the $T_{C}$ is weak in $\mathrm{ZnNNi}_{3}$. Compared with $\mathrm{MgCNi}_{3}$, a significantly reduced $N\left(E_{F}\right)$ was theoretically observed in $\mathrm{ZnNNi}_{3}$ [42], which accounts for the lower $T_{C}$ in $\mathrm{ZnNNi}_{3}$ than in $\mathrm{MgCNi}_{3}$. The $\mathrm{CdNNi}_{3}$ and $\mathrm{InNNi}_{3}$ were also successfully synthesized by the same authors of [41] but neither is superconducting [43]. The $\gamma$ value is $12 \mathrm{~mJ} /\left(\mathrm{mol} \mathrm{K}^{2}\right)$ and $8 \mathrm{~mJ} /\left(\mathrm{mol} \mathrm{K}^{2}\right)$ for $\mathrm{CdNNi}_{3}$ and $\mathrm{InNNi}_{3}$, respectively, smaller than that for $\mathrm{ZnNNi}_{3}$. However, the temperature-independent magnetic susceptibility $\chi_{0}$ for $\mathrm{CdNNi}_{3}$ and $\mathrm{InNNi}_{3}$ is larger than that of $\mathrm{ZnNNi}_{3}$, indicative of an enhanced contribution from the FM correlations in the former two compounds. It shows by theoretical calculations that the $N\left(E_{F}\right)$ for $\mathrm{CdNNi}_{3}$ is comparable with that for $\mathrm{ZnNNi}_{3}$ [44], but the $N\left(E_{F}\right)$ is much reduced in $\mathrm{InNNi}_{3}$ [45]. Assuming that the e-p coupling is comparable in $\mathrm{CdNNi}_{3}$ and $\mathrm{ZnNNi}_{3}$, it is possible to observe superconductivity in $\mathrm{CdNNi}_{3}$ in case the FM correlations can be well suppressed. Very recently, He et al. reported two series of doped $\mathrm{CdNNi}_{3}$, that is, $\mathrm{Cd}_{1-x} \operatorname{In}_{x} \mathrm{NNi}_{3}(0 \leq x \leq 0.2)$ and $\mathrm{Cd}_{1-x} \mathrm{Cu}_{x} \mathrm{NNi}_{3}(0 \leq$ $x \leq 0.2$ ) [46]. These compounds show metallic resistivity and exhibit a Fermi liquid behavior at low temperatures. No superconductivity was found down to $2 \mathrm{~K}$. However, all samples exhibit very soft and weak ferromagnetism, in contrast to the PM behavior for $\mathrm{CdNNi}_{3}$ reported previously by Uehara et al. [43].

Compared with the carbides $\mathrm{ACNi}_{3}$ and nitrides $\mathrm{ANNi}_{3}$, little attention has been paid to the borides $\mathrm{ABNi}_{3}$. To the best of our knowledge, $\mathrm{ScB}_{0.5} \mathrm{Ni}_{3}$ [47] is the only boron based Ni-based antiprovskite compound with its physical properties reported in the literatures. It shows a Pauli PM behavior without any superconducting signals observed down to $2 \mathrm{~K}$. We tried to synthesize $\mathrm{ABNi}_{3}(\mathrm{~A}=\mathrm{Al}, \mathrm{Ga}$, In, and so on) samples by solid state reaction [48]. The pure sample of $\mathrm{InBNi}_{3}$ with the antiperovskite structure (lattice constant $a=3.795 \AA$ ) was successfully synthesized and structural, magnetic, transport properties, and specific heat measurements performed. No superconductivity appears down to the lowest temperature by electric and magnetic measurements $(5 \mathrm{~K})$ as shown in Figure 5(a). The magnetization $\chi(T)$ takes a typical Pauli PM behavior with a very small contribution from the FM spin fluctuations. As shown in the inset of Figure 5(b), the low-temperature specific heat data, plotted as $C(T) / T$ versus $T^{2}$, can be well fitted using the following formula, $C(T) / T=\gamma+$ $\beta T^{2}$, where $\gamma$ is the Sommerfeld constant for electronic 


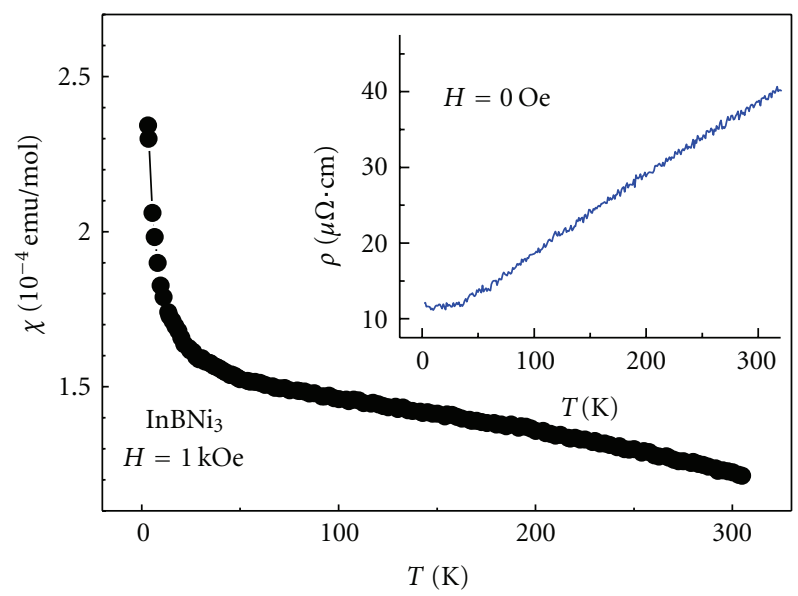

(a)

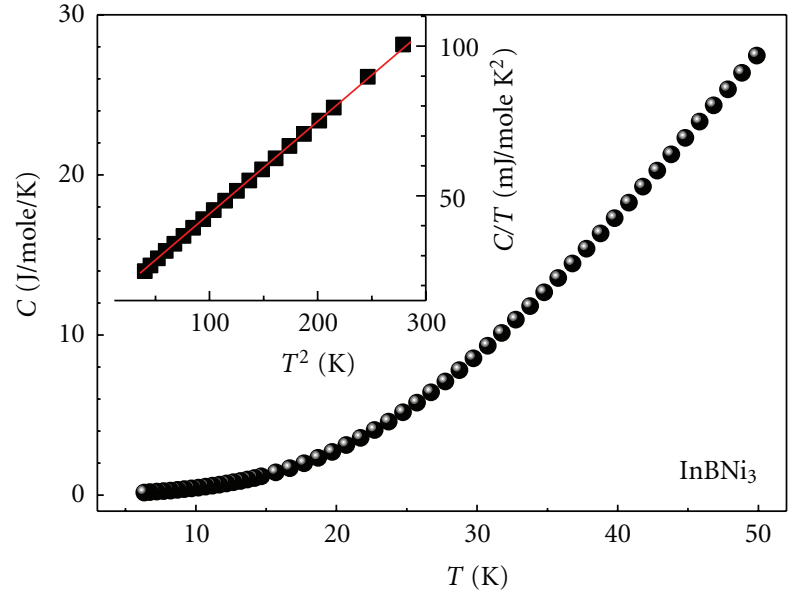

(b)

Figure 5: (Color online) The physical properties for $\operatorname{InBNi}_{3}$. (a) dc magnetic susceptibility $\chi(T)$ at $H=1$ kOe. Inset shows the temperature dependence of resistivity measure at zero field. (b) Specific heat as a function of temperature. Inset shows a linear fit of $C(T) / T$ versus $T^{2}$ below $15 \mathrm{~K}$.

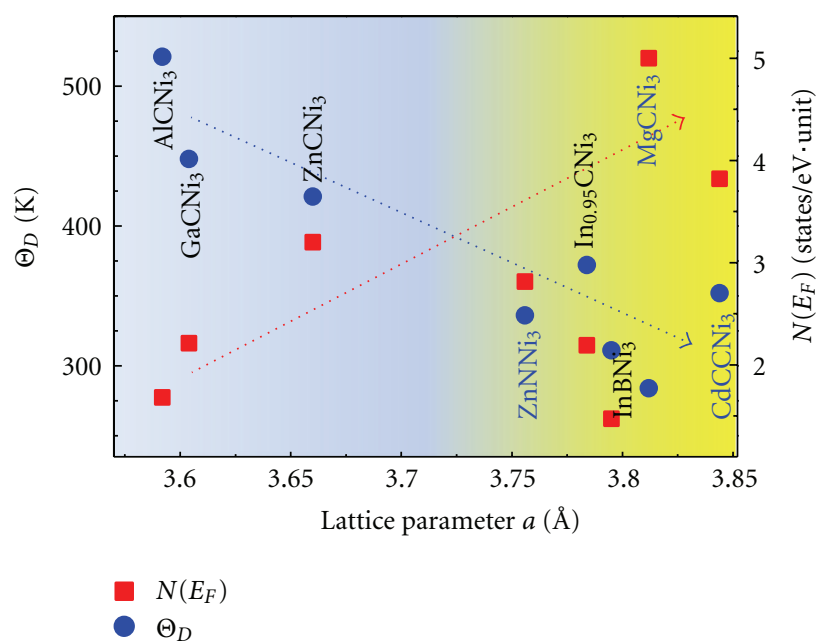

Figure 6: (Color online) The Debye temperature, $\Theta_{D}$, and the DOS at $E_{F}, N\left(E_{F}\right)$ as a function of the lattice parameter $a$ for Ni-based antiperovskite compounds. The crossing dashed lines indicate the trends of $\Theta_{D}$ (downwards) and $N\left(E_{F}\right)$ (upwards) with increasing the lattice constant $a$. The $\Theta_{D}$ values are derived from specific heat data reported in $[26,29,31,32,41]$. The $N\left(E_{F}\right)$ values are from theoretical calculations where corresponding calculated lattice constant is the closet to the experimental ones $[4,28,30,37,39,40$, 42].

contribution and the second term represents the phonon contribution according to the Debye approximation $[31,32]$. The fitted values of $\gamma$ and $\beta$ are equal to $11.33 \mathrm{~mJ} /\left(\mathrm{mol} \mathrm{K}^{2}\right)$ and $0.32 \mathrm{~mJ} /\left(\mathrm{mol} \mathrm{K}^{4}\right)$, respectively. The Debye temperature $\Theta_{D}$ is estimated to be $311 \mathrm{~K}$ according to the formula, $\Theta_{D}=$ $\left(n \times 1.944 \times 10^{6} / \beta\right)^{1 / 3}$, where $n$ is the number of atoms in a unit cell. The Wilson ration is estimated to be 0.93 , very close to the free electrons, indicating weak FM correlations or spin fluctuations in $\mathrm{InBNi}_{3}$. The value of $\gamma$ is smaller than the superconducting compounds in Table 1. So the e-p coupling is weak in this compound, accounting for the disappearance of superconductivity. Theoretically, the $N\left(E_{F}\right)$ is only 1.47 states $\mathrm{eV}^{-1} / \mathrm{f} \cdot \mathrm{u}$ [49], consistent with the observed small $\gamma$. Besides, the authors predicted that introduction of holes into $\mathrm{InBNi}_{3}$ could make it superconducting, which has not been proved experimentally yet. Theoretically, it is predicted that $\mathrm{AlBNi}_{3}$ is candidate for studying unconventional superconductivity, which has not been tested experimentally either [50].

\section{A Universal Phase Diagram}

Thanks to the systematic studies in the past, it is possible to draw a uniform picture of the properties for the Ni-based antiperovskite compounds, thus to shed light on the unique superconductivity in $\mathrm{MgCNi}_{3}$. The Debye temperature $\Theta_{D}$ obtained from specific heat measurements, the calculated density of state at Fermi level $N\left(E_{F}\right)$ available in the published literatures are plotted as a function the lattice constant, as shown in Figure 6. Two main trends can be found, (1) the $\Theta_{D}$ increases approximately as the lattice constant is reduced. (2) The shrinkage of lattice constant reduces the $N\left(E_{F}\right)$, which is more scattered than $\Theta_{D}$ though.

The evolution of $\Theta_{D}$ derived from experimental specific heat data with lattice constant can be understood as follows: the lattice contraction leads to the hardening of phonon mode, thus an increase of Debye temperature [29]. There exists a strong hybridization between $\mathrm{X} 2 \mathrm{p}$ and $\mathrm{Ni} 3 \mathrm{~d}$ orbitals $[4,5,16,25,30,36,37]$, playing important roles in determining the physical properties. The decrease of lattice constant reduces the $\mathrm{Ni}-\mathrm{C}$ bond length, thus enhances the hybridization, leading to a decreased $N\left(E_{F}\right)$. It is more general that the DOS is inversely proportional to the band 
width $W$. for a cubic solid, the band width is related with the lattice constant $a$ by the expression $W \sim 1 / a^{3}$ [29]. Therefore, the decrease of lattice constant will increase the band width, leading to a reduction of $N\left(E_{F}\right)$. In addition, the theoretical calculations show there is a peak structure in the DOS below the $E_{F}$ for all Ni-based antiperovskite compounds $\mathrm{AXNi}_{3}$. For carbide compounds $\mathrm{ACNi}_{3}(\mathrm{~A}=\mathrm{Al}$, $\mathrm{Ga}$, In) or $\mathrm{ZnNNi}_{3}$ that has more electrons than $\mathrm{MgCNi}_{3}$, could be interpreted as electron-doped $\mathrm{MgCNi}_{3}$, resulting in a downward shift of the position of the peak in the DOS from the $E_{F}$, consequently a reduced $N\left(E_{F}\right)$ [39]. In a word, the $N\left(E_{F}\right)$ is expected to increase as the lattice expands. It is basically followed by many compounds as shown in Figure 6. However, the real case may be too complex to be attributed to the above models. One example is $\mathrm{InBNi}_{3}$ whose $N\left(E_{F}\right)$ is extremely lower than expected. It is probably because the $\mathrm{B}$ $2 \mathrm{p}$ state in borides hybridizes with $\mathrm{Ni} 3 \mathrm{~d}$ state more than the C 2p state in carbides $\mathrm{ACNi}_{3}$ [51].

For a BCS theory, the e-p coupling constant can be estimated by the McMillan's formula [32], $\lambda_{p h}=$ $\left[N\left(E_{F}\right)\left\langle I^{2}\right\rangle / M\langle\omega\rangle\right]$, where $\left\langle I^{2}\right\rangle$ is the averaged electron-ion matrix element squared, $M$ is an atomic mass, and $\left\langle\omega^{2}\right\rangle$ the averaged phonon frequency proportional to Debye temperature $\Theta_{D}$. Therefore, a combination of a large $N\left(E_{F}\right)$ and small $\Theta_{D}$ will lead to a strong e-p coupling, consequently a BCS-like superconductor. The Ni-based antiperovskite compounds seem to obey this raw. All discovered superconductors locate on the right side of the map in Figure 6, where the $\Theta_{D}$ is relatively small, but the $N\left(E_{F}\right)$ is relatively large. For instance, $\mathrm{MgCNi}_{3}$ which shows the highest $T_{C}$ has the largest $N\left(E_{F}\right)$ and smallest $\Theta_{D}$. Figure 6 also suggests that the superconductivity observed in the Ni-based antiperovskite compounds is predominantly $s$-wave BCS type mediated by e-p coupling, though the other contributions, for example, from spin fluctuations, may not be excluded. We note that the $\Theta_{D}$ for $\mathrm{MgCNi}_{3}$ in the figure is from polycrystalline sample [27], while the value deduced from resistivity for single crystal $\mathrm{MgCNi}_{3}$ is surprisingly small $(132 \mathrm{~K})$ [18]. There is no clear trend for the relation between number of the valence electrons and $N\left(E_{F}\right)$ or $\Theta_{D}$. Regardless of this, the phase diagram in Figure 6 provides a clue for searching new superconductors in Ni-based antiperovskite compound $\mathrm{AXNi}_{3}$, namely, the compounds with large lattice constant may be superconducting in terms of the BCS scenario.

\section{Future Outlook}

In the future, the following works are worthy to be done.

(1) $\mathrm{New} \mathrm{MgCNi}_{3}$ single crystals with ideal 1:1:3 stoichiometry would finally close the long-time debate on the mechanism of superconductivity.

(2) In order to clarify the divergences among the experimental and theoretical results for the Ni-based antiperovksite compounds other than $\mathrm{MgCNi}_{3}$, more extensive investigations on single crystal samples are desirable. The growth of single crystal $\mathrm{AXNi}_{3}$ is a challenge. The successful growth of $\mathrm{MgCNi}_{3}$ single crystals would help, because the application of high pressure during heating can improve the solubility of carbon and suppresses the volatility of magnesium.

(3) The $\mathrm{AXNi}_{3}$ materials may serve as a platform for studying quantum critical phenomena (QCP) and quantum phase transitions (QPT) in simple material systems with three-dimensional cubic structure and none "f" elements. Previously, the QCP and QPT have been extensively studied in some "unique" systems [52], such as heavy fermions with "f" elements, magnetic systems with spin frustration, and so on. Taking the advantages of the single crystal samples, the possible quantum phase transitions can be explored in some $\mathrm{AXNi}_{3}$, such as $\mathrm{AlCNi}_{3}, \mathrm{GaCNi}_{3}$, and $\mathrm{CdCNi}_{3}$, driven by chemical alloying, external pressure, or magnetic field.

(4) It is interesting to explore new superconductors with antiperovskite structure based the clues mentioned, for example, large lattice constant may favor BCS superconductivity. This clue may work for the antiperovskite compounds based on other $3 \mathrm{~d}$ elements, such as $\mathrm{Ti}$ and Sc. The discovery of new superconductors can always cheer the superconductor society.

\section{Conclusion}

We summarized the recent progress for Ni-based antiperovskite compounds closely related to the superconducting $\mathrm{MgCNi}_{3}$. A universal phase diagram is presented based on the published data, which would help design new superconductors with the antiperovskite structure. The synthesis and characterization on single crystals are desirable for future study in order to eliminate the divergences made by different authors or between the theoretical and experimental result.

\section{Acknowledgments}

This work was supported by the National Key Basic Research under Contract no. 2011CBA00111 and the National Natural Science Foundation of China under Contract nos. 50701042, 11174295, 51001094, 51171177, and 91222109.

\section{References}

[1] J. G. Bednorz and K. A. Müller, "Possible high $T_{c}$ superconductivity in the Ba-La-Cu-O system," Zeitschrift für Physik B, vol. 64, no. 2, pp. 189-193, 1986.

[2] T. He, Q. Huang, A. P. Ramirez et al., "Superconductivity in the non-oxide perovskite $\mathrm{MgCNi}_{3}$," Nature, vol. 411, no. 6833, pp. 54-56, 2001.

[3] H. Rosner, R. Weht, M. D. Johannes, W. E. Pickett, and E. Tosatti, "Superconductivity near ferromagnetism in $\mathrm{MgCNi}_{3}$ " Physical Review Letters, vol. 88, no. 2, Article ID 027001, pp. 270011-270014, 2002.

[4] D. J. Singh and I. I. Mazin, "Superconductivity and electronic structure of perovskite $\mathrm{MgCNi}_{3}$," Physical Review B, vol. 64, no. 14, Article ID 140507, pp. 1405071-1405074, 2001. 
[5] J. H. Shim, S. K. Kwon, and B. I. Min, "Electronic structures of antiperovskite superconductors $\mathrm{Mg}_{x} \mathrm{Ni}_{3}(\mathrm{X}=\mathrm{B}, \mathrm{C}$, and N)," Physical Review B, vol. 64, no. 18, Article ID 180510, pp. 1805101-1805104, 2001.

[6] P. M. Singer, T. Imai, T. He, M. A. Hayward, and R. J. Cava, " ${ }^{13} \mathrm{C}$ NMR investigation of the superconductor $\mathrm{MgCNi}_{3}$ up to $800 \mathrm{~K}$," Physical Review Letters, vol. 87, no. 25, Article ID 257601, pp. 257601/1-257601/4, 2001.

[7] R. Prozorov, A. Snezhko, T. He, and R. J. Cava, "Evidence for unconventional superconductivity in the nonoxide perovskite $\mathrm{MgCNi}_{3}$ from penetration depth measurements," Physical Review B, vol. 68, no. 18, Article ID 180502, pp. 18050211805024, 2003.

[8] X. F. Lu, L. Shan, Z. Wang et al., "Evidence for s-wave pairing from measurement of the lower critical field in $\mathrm{MgCNi}_{3}$," Physics Review B, vol. 71, no. 18, Article ID 174511, 2005.

[9] D. P. Young, M. Moldovan, and P. W. Adams, "Scaling behavior of the critical current density in $\mathrm{MgCNi}_{3}$ microfibers," Physical Review B, vol. 70, no. 6, Article ID 064508, pp. 064508-5, 2004.

[10] Z. Q. Mao, M. M. Rosario, K. D. Nelson et al., "Experimental determination of superconducting parameters for the intermetallic perovskite superconductor $\mathrm{MgCNi}_{3}$," Physical Review B, vol. 67, no. 9, Article ID 094502, pp. 945021-945026, 2003.

[11] L. Shan, H. J. Tao, H. Gao et al., "s-wave pairing in $\mathrm{MgCNi}_{3}$ revealed by point contact tunneling," Physical Review B, vol. 68, no. 14, Article ID 144510, pp. 1445101-1445105, 2003.

[12] T. Klimczuk and R. J. Cava, "Carbon isotope effect in superconducting $\mathrm{MgCNi}_{3}$," Physical Review B, vol. 70, no. 21, Article ID 212514, pp. 1-3, 2004.

[13] J.-Y. Lin, P. L. Ho, H. L. Huang et al., "BCS-like superconductivity in $\mathrm{MgCNi}_{3}$," Physical Review B, vol. 67, no. 5, Article ID 052501, pp. 525011-525014, 2003.

[14] A. Wälte, G. Fuchs, K. H. Müller et al., "Evidence for strong electron-phonon coupling in $\mathrm{MgCNi}_{3}$," Physical Review B, vol. 70, no. 17, Article ID 174503, pp. 1-18, 2004.

[15] G. J. MacDougall, R. J. Cava, S. J. Kim et al., "Muon spin rotation study of $\mathrm{MgCNi}_{3}$," Physica B, vol. 374-375, pp. 263266, 2006.

[16] S. Mollah, "The physics of the non-oxide perovskite superconductor $\mathrm{MgCNi}_{3}$," Journal of Physics Condensed Matter, vol. 16, no. 43, pp. R1237-R1276, 2004.

[17] H.-S. Lee, D. J. Jang, H. G. Lee, S. I. Lee, S. M. Choi, and C. J. Kim, "Growth of single crystals of $\mathrm{MgCNi}_{3}$," Advanced Materials, vol. 19, no. 14, pp. 1807-1809, 2007.

[18] H.-S. Lee, D. J. Jang, H. G. Lee, W. Kang, M. H. Cho, and S. I. Lee, "Evidence of conventional superconductivity in singlecrystalline MgCNi 3," Journal of Physics Condensed Matter, vol. 20, no. 25, Article ID 255222, 2008.

[19] J. Kačmarcík, Z. Pribulov, C. Marcenat et al., "Specific heat of superconducting $\mathrm{MgCNi}_{3}$ single crystals," Journal of Physics, vol. 150, no. 5, Article ID 052087, 2009.

[20] Z. Pribulová, J. Kačmarčík, C. Marcenat et al., "Superconducting energy gap in $\mathrm{MgCNi}_{3}$ single crystals: Point-contact spectroscopy and specific-heat measurements," Physical Review B, vol. 83, no. 10, Article ID 104511, 2011.

[21] P. Diener, P. Rodière, T. Klein et al., " $s$-wave superconductivity probed by measuring magnetic penetration depth and lower critical field of $\mathrm{MgCNi}_{3}$ single crystals," Physical Review B, vol. 79, no. 22, Article ID 220508, 2009.

[22] H. Hong, M. Upton, A. H. Said et al., "Phonon dispersions and anomalies of $\mathrm{MgCNi}_{3}$ single-crystal superconductors determined by inelastic x-ray scattering," Physical Review B, vol. 82, no. 13, Article ID 134535, 2010.
[23] P. K. Jha, S. D. Gupta, and S. K. Gupta, "Puzzling phonon dispersion curves and vibrational mode instability in superconducting $\mathrm{MgCNi}_{3}$," AIP Advances, vol. 2, no. 2, Article ID 022120, 2012.

[24] D.-J. Jang, H. S. Lee, H. G. Lee, M. H. Cho, and S. I. Lee, "Collapse of the peak effect due to ac-induced flux creep in an isotropic vortex system of $\mathrm{MgCNi}_{3}$ single crystals," Physical Review Letters, vol. 103, no. 4, Article ID 047003, 2009.

[25] I. R. Shein and A. L. Ivanovskii, "Electronic and elastic properties of non-oxide anti-perovskites from first principles: Superconducting $\mathrm{CdCNi}_{3}$ in comparison with magnetic $\mathrm{InCNi}_{3}$," Physics Review B, vol. 77, no. 10, Article ID 104101, 2008.

[26] P. Tong, Y. P. Sun, X. B. Zhu, and W. H. Song, "Synthesis and physical properties of antiperovskite-type compound In $0.95 \mathrm{CNiM}_{3}$," Solid State Communications, vol. 141, no. 6, pp. 336-340, 2007.

[27] M. Uehara, T. Yamazaki, T. Kôri, T. Kashida, Y. Kimishima, and I. Hase, "Superconducting properties of $\mathrm{CdCNi}_{3}$," Journal of the Physical Society of Japan, vol. 76, no. 3, Article ID 034714, 2007.

[28] S. Bağc1, S. Duman, H. M. Tütüncü, and G. P. Srivastava, "Ground state, phonon spectrum, and superconducting properties of the nonoxide perovskite $\mathrm{CdCNi}_{3}$," Physics Review B, vol. 78, no. 17, Article ID 174504, 2008.

[29] M. S. Park, J. S. Giim, S. H. Park, Y. W. Lee, S. I. Lee, and E. J. Choi, "Physical properties of $\mathrm{ZnCNi}_{3}$ : Comparison with superconducting $\mathrm{MgCNi}_{3}$," Superconductor Science and Technology, vol. 17, no. 2, pp. 274-277, 2004.

[30] M. D. Johannes and W. E. Pickett, "Electronic structure of $\mathrm{ZnCNi}_{3}$," Physical Review B, vol. 70, no. 6, pp. 060507-4, 2004.

[31] P. Tong, Y. P. Sun, X. B. Zhu, and W. H. Song, "Strong electronelectron correlation in the antiperovskite compound $\mathrm{GaCNi}_{3}$," Physical Review B, vol. 73, no. 24, Article ID 245106, 2006.

[32] P. Tong, Y. P. Sun, X. B. Zhu, and W. H. Song, "Strong spin fluctuations and possible non-Fermi-liquid behavior in $\mathrm{AlCNi}_{3}$," Physics Review B, vol. 74, no. 22, Article ID 224416, 2006.

[33] B. Chen, C. Michioka, Y. Itoh, and K. Yoshimura, "Synthesis and magnetic properties of $\mathrm{Ni}_{3} \mathrm{AlC}_{x}$," Journal of the Physical Society of Japan, vol. 77, no. 10, Article ID 103708, 2008.

[34] B. Chen, H. Ohta, C. Michioka, Y. Itoh, and K. Yoshimura, "27 Al NMR studies of itinerant electron ferromagnetic $\mathrm{Ni}_{3}$ $\mathrm{AlC}_{x}$," Physical Review B, vol. 81, no. 13, Article ID 134416, 2010.

[35] A. F. Dong, G. C. Che, W. W. Huang, S. L. Jia, H. Chen, and Z. X. Zhao, "Synthesis and physical properties of $\mathrm{AlCNi}_{3}$," Physica C, vol. 422, no. 1-2, pp. 65-69, 2005.

[36] C. M. I. Okoye, "Theoretical investigation of electronic structure and optical properties of paramagnetic non-oxide perovskite $\mathrm{AlCNi}_{3}$," Solid State Communications, vol. 136, no. 11-12, pp. 605-610, 2005.

[37] M. Sieberer, P. Mohn, and J. Redinger, "Role of carbon in $\mathrm{AlCNi}_{3}$ and $\mathrm{GaCNi}_{3}$ : a density functional theory study," Physics Review B, vol. 75, no. 2, Article ID 024431, 2007.

[38] G. H. Zhong, J. L. Wang, Z. Zeng, X. H. Zheng, and H. Q. $\mathrm{Lin}$, "Induced effects by the substitution of $\mathrm{Mg}$ in $\mathrm{MgCNi}_{3}$," Journal of Applied Physics, vol. 101, no. 9, Article ID 09G520, 2007.

[39] F. Boutaiba, A. Zaoui, and M. Ferhat, "Ground state analysis of $\mathrm{XCNi}_{3}(\mathrm{X}=\mathrm{Mg}, \mathrm{Zn}$, and $\mathrm{Ga}$ ) from first-principles," Physica $B$, vol. 406, no. 2, pp. 265-269, 2011. 
[40] S. Q. Wu, Z. F. Hou, and Z. Z. Zhu, "Electronic structure and magnetic state of $\mathrm{InCNi}_{3}$," Physica B, vol. 403, no. 23-24, pp. 4232-4235, 2008.

[41] M. Uehara, A. Uehara, K. Kozawa, and Y. Kimishima, "New antiperovskite-type superconductor $\mathrm{ZnNyNi}_{3}$," Journal of the Physical Society of Japan, vol. 78, no. 3, pp. 0337021-0337024, 2009.

[42] I. R. Shein, V. V. Bannikov, and A. L. Ivanovskii, "Elastic and electronic properties of the new perovskite-like superconductor $\mathrm{ZnNNi}_{3}$ in comparison with $\mathrm{MgCNi}_{3}$," Physica Status Solidi B, vol. 247, no. 1, pp. 72-76, 2010.

[43] M. Uehara, A. Uehara, K. Kozawa, T. Yamazaki, and Y. Kimishima, "New antiperovskite superconductor $\mathrm{ZnNNi}_{3}$, and related compounds $\mathrm{CdNNi}_{3}$ and $\mathrm{InNNi}_{3}$," Physica $C$, vol. 470, no. 1, pp. S688-S690, 2010.

[44] C. Li, W. G. Chen, F. Wang et al., "First-principles investigation of mechanical and electronic properties of $\mathrm{MNNi}_{3}(\mathrm{M}=\mathrm{Zn}$, Mg, or Cd)," Journal of Applied Physics, vol. 105, no. 12, Article ID 123921, 2009.

[45] Z. F. Hou, "Elastic properties and electronic structures of antiperovskite-type $\mathrm{InNCO}_{3}$ and $\mathrm{InNNi}_{3}$," Solid State Communications, vol. 150, no. 39-40, pp. 1874-1879, 2010.

[46] B. He, C. Dong, L. H. Yang, L. H. Ge, L. B. Mu, and X. C. Chen, "Preparation and the physical properties of antiperovskite-type compounds $\mathrm{Cd}_{1-x} \mathrm{In}_{x} \mathrm{NNi}_{3}(0 \leq x \leq 0.2)$ and $\mathrm{Cd}_{1-y} \mathrm{Cu}_{y} \mathrm{NNi}_{3}(0 \leq y \leq 0.2)$," Chinese Physics B, vol. 21, no. 4, Article ID 047401, 2012.

[47] T. Shishido, K. Kudou, T. Sasaki et al., "Search for perovskitetype new borides in the Sc-TM-B (TM = Ti, V, Cr, Mn, Fe, Co, and Ni) systems," Journal of Alloys and Compounds, vol. 383, no. 1-2, pp. 294-297, 2004.

[48] P. Tong, Study on the physical properties of nickel based antiperovskite compounds [Ph.D. thesis], 2007.

[49] I. R. Shein, A. L. Ivanovskii, and N. I. Medvedeva, "Electronic structure of the new $\mathrm{MgCNi}_{3}$ superconductor and related intermetallic compounds," JETP Letters, vol. 74, no. 2, pp. 122-127, 2001.

[50] I. Hase, " $\mathrm{Ni}_{3} \mathrm{AlB}$ : A bridge between superconductivity and ferromagnetism," Physics Review B, vol. 70, no. 3, Article ID 033105, 2004.

[51] I. Hase, "Electronic structure of $\mathrm{Ni}_{3} \mathrm{AlX}_{y}(\mathrm{X}=\mathrm{B}, \mathrm{C}, \mathrm{H} ; 0<y<$ 1)," Materials Transactions, vol. 47, no. 3, pp. 475-477, 2006.

[52] H. V. Löhneysen, A. Rosch, M. Vojta, and P. Wölfle, "Fermiliquid instabilities at magnetic quantum phase transitions," Reviews of Modern Physics, vol. 79, no. 3, pp. 1015-1075, 2007. 

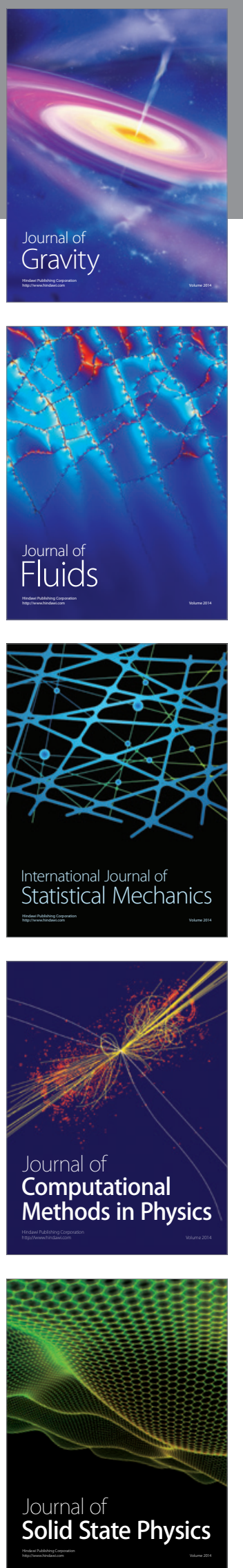

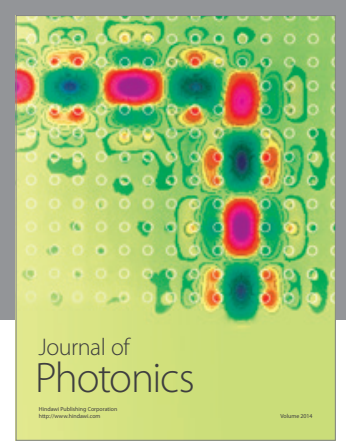

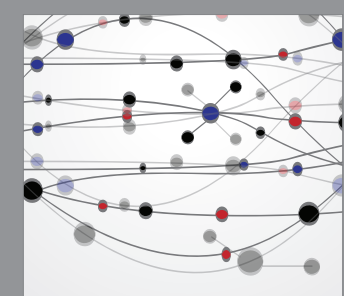

The Scientific World Journal
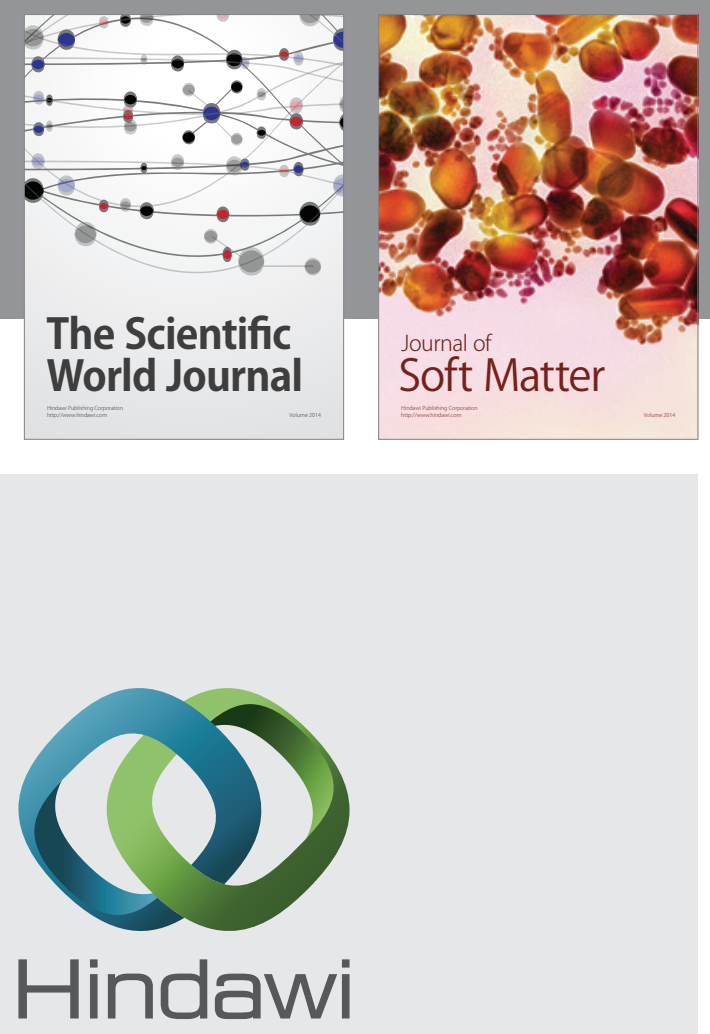

Submit your manuscripts at

http://www.hindawi.com
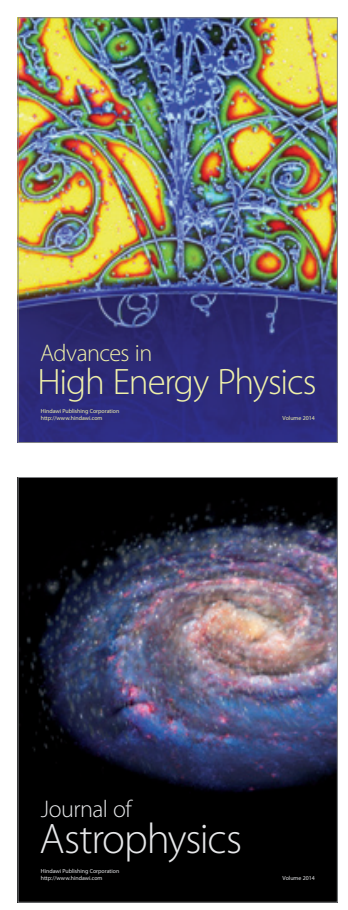
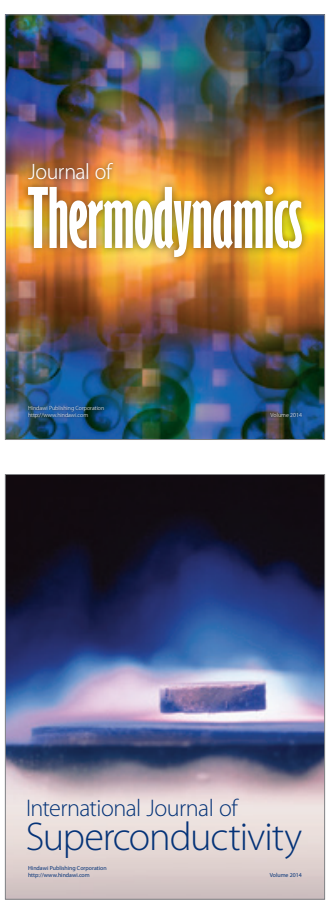
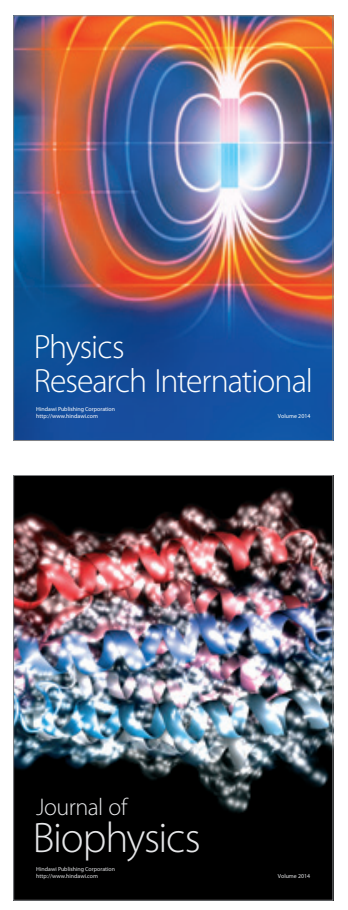
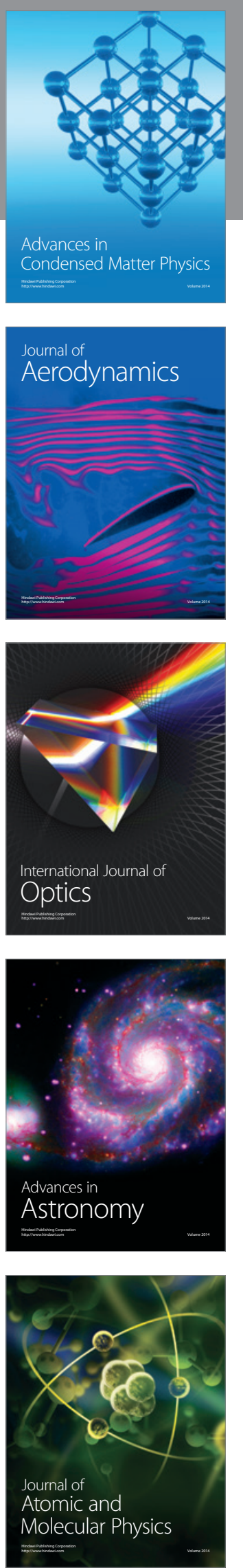